\title{
Traffic Modeling in Mobile Communication Networks
}

\author{
Osahenvemwen O.A. \\ Department of Electrical and \\ Electronics \\ Faculty of Engineering and \\ Technology. \\ Ambrose Alli, University. \\ Ekpoma, Edo State, Nigeria.
}

\author{
EDEKO F.O. \\ Department of Electrical and \\ Electronics \\ Faculty of Engineering and \\ Technology. \\ University of Benin. \\ Benin, Edo State, Nigeria
}

\author{
EMAGBETERE J. \\ Department of Elect/Elect \\ Engineering \\ Faculty of Engineering and \\ Technology. \\ University of Benin \\ Benin City, Edo State.
}

\begin{abstract}
This paper is focused on traffic modeling in Mobile Communication networks. This research is aimed at developing a traffic models that will predict a blocking probability for voice calls and handover calls blocking probability in mobile communication networks (GSM). The high number of block calls experience in mobile network, especially during the Busy Hour $(\mathrm{BH})$ has leads to poor Quality of Service (QOS) delivering in mobile network. These block calls experience in mobile network should be reduced to a certain low values $(2 \%$ value in line with $\mathrm{NCC}$ recommended standard), to ensure good QOS. The developed traffic models are focused on new voice calls within the cell, handover calls in and out of a cell. The developed traffic models are designed based on the numbers of channels, partition into two segments in a cell network. The cell technology is homogenous in nature; therefore it is applicable to the entire mobile communication system. The analytical method is deployed, and the collection of traffic data with equipment know as the Operation and Maintenance Center (OMC-counter) which is in built in the mobile communication network is used. The OMC-counter runs on Linux operation software, which helps to capture the number of arrival calls and service time in a specified interval. The arrival rate is assumed to be Poisson and the service time is also, assumed to be exponentially distributed and independence identical distributed. These parameters are used in the development of the traffic models. The developed traffic models are blocking probability for voice calls and handover blocking probability for handover calls. These developed (propose) traffic models are validated using MATLAB (version 7.6.3. 325) program and compared with the conventional Erlang B for accuracy. These traffic models are used to manage the block calls experience in the mobile network. Also used in a balance relationship between cost incurred in mobile communication by operators and service render to the mobile communication subscribers.
\end{abstract}

Keywords: Arrival rate, service time, exponential distribution, traffic model and traffic load in erlang.

\section{INTRODUTION}

The mobile communication network is faced with serious challenges to ensure good Quality of Service (QOS).The Good QOS in mobile communication network is necessary at this, presence economic situation of global economic meltdown and competitive business environment with low tariff. In order to survive in this present economic situation, the network operators must put into consideration the effective use of their available resources (channels), which leads to effective network design and network planning.
These major parameters used for effective network design and network planning are blocking probability, handover blocking probability, Utilization etc. The block probability is used, to control the number of block calls experience in the mobile network and this block calls arises from lack of network capacity (transmission channels) to accommodate or carry all the calls at a particular point in time [7],[9].The blocking probability is determined from the number of available channels and traffic load in erlang. In evaluating the performance of handover blocking probability the following parameters must be consider as follows, handover rate, dropping probability, handover probability, call holding time and channel holding time[16][8].The calls dropping probability, its the probability that calls that originally (initially) granted access to the network channels (switches), but due to technical error(such are Electromagnetic causes, irregular user behavior etc) the calls are truncated (forced termination) during conversation. The evaluation of drop calls is referred to as handover blocking probability. The handover rate is used to estimate the handover traffic arrival rate, which is an input parameter in the determination of calls blocking probability. The channel holding time is determined by the cell residence time (cell dwell time).The cell residence is affected by the subscriber mobility, the geographic situation and the types of channel allocation schemes used (or other factors such as fading). Channel holding time, is defined as the time a Mobile Station (MS) remains in the same cell during a call, while call holding time is the total call duration time. The total call duration time include the time when MS move from a cell to another new cell, this process is called handover process[15],[14].

In order, to estimate the volume of offered traffic load in erlang place on mobile communication network capacity and the proportion number of block calls (subscribers) in the system, will need to deploy a model.

From literature review, it is observed that queuing model have been successfully applied to area such a capacity planning and performance analysis [16],[12] .

A model is a mathematical expression or diagram or algorithms that represent traffic characteristics [17]. This traffic model show the relationship between these components, channels resource $(\mathrm{V})$, traffic load in erlang $(\mathrm{A})$ and blocking probabilities $\left(\mathrm{P}_{\mathrm{b}}\right)$. The relationship between traffic loads and services state, show that as the offered traffic load increases and service render to subscribers decline in a constant capacity (channels)[5]. Therefore there is need to predicted accurate traffic load in line with adequate capacity (channels) in mobile communication network to minimize block calls experience by subscribers [3],[2]. The existing traffic model are Binomial, Poisson, Erlang B, Erlang C etc are used in network planning and network design, to 
minimized block calls experience by subscribers in the network [6]. The traditional traffic models (like Erlang B formula) are not adequate to this presence mobile communication network, because with the inclusion of mobility and data service into the mobile network. Therefore, there is need for adequate traffic models that will incorporate the subscribers handover calls (mobility) and data service into the voice call framework.

\section{Modeling of a telecommunication system}

In model of telecommunication system, it most involves the entire mobile communication network section. The modeling of telecommunication system is divided into three main elements in Fig1; these are traffic user (demand), structure (hardware) and operational strategy (software) [6],[12].

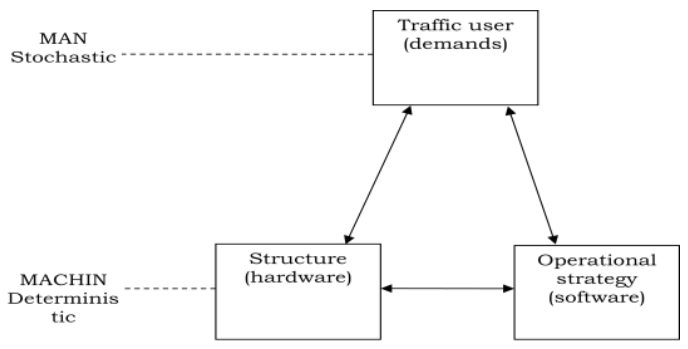

Fig. 1: The telecommunication system

In Fig1 telecommunication system, is made up of two major statistical components, these are, the traffic usage (traffic demands) which is Random in nature and deterministic (machine) components are, structure (hardware) and operational strategy [6].

Traffic is trigger by the subscribers, and it's made-up of arrival rate and service time shown in Fig 2.

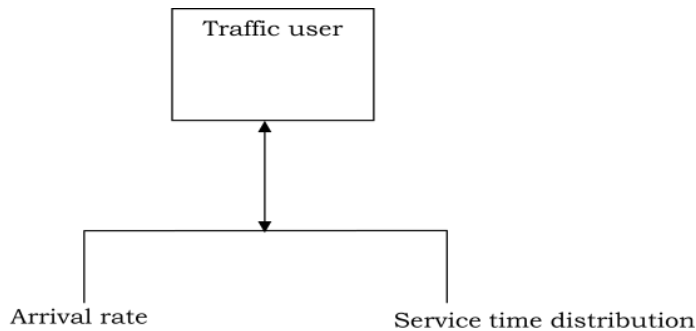

Fig 2. The traffic random component

Traffic user demands are random in nature, which means that subscribers generate calls in a random process. Random process is one in which one or more quantities vary with time in such a way that the instantaneous values of quantities are not determined precisely but are predictable with certain probability. These quantities are known as random variables [12]. Traffic user demands components are the arrival rate and service time distribution which is model by statistical properties [6],[1],[10].

Structure it's consider as the hardware in the system, example is identical channels (servers, trunks, slots) working in parallel. This is also referred to as homogenous group.

Strategy it's referred to as operational software, example are queue discipline, the order or manner in which customers are selected for service [5].Example of queues discipline are as follows, First-Come-First-Served (FCFS), Last-Come-First Served (LCFS), Service in Random Order (SIRO) and Priority service. A call arriving at the system is accepted for service if at least one channel is idle. A call can not be block if the system is free, except all channels are busy in the system [11].

These are basic parameters consider for queuing model of traffic in mobile communication network, as follows [17][13].

1. Arrival process(inter- arrival distribution)

2. The service time distribution

3. Number of parallel server ( finite or infinite)

4. Capacity of the system (full or restricted availability)

5. Size of the population for the system

6. Queue discipline

From research work, the exist queue models do take different format such as $\mathrm{M} / \mathrm{M} / 1, \mathrm{M} / \mathrm{G} / 1, \mathrm{G} / \mathrm{M} / 1$ and $\mathrm{G} / \mathrm{G} / 1$.In all these models, M/M/1 model is the most disciplined and have the means of evaluating the network performance to estimate the queuing parameters, i.e. "Queue length" and "waiting time". This same queue model, assume exponential distribution in traffic situation [11], [16], [14].

\section{METHODOLOGY}

An analytical approach is deployed to determine a traffic model, assuming a certain number of channels in cell system, which is also homogenous in the entire mobile communication network. The cell is assumed to contain a certain number of channels. The queuing theory is deployed as model technique, assuming the calls enter the system in an orderly sequence. The developed traffic model is design based on the arrival rate, holding time, which is statistical distributed among subscriber making calls within the cell and handover calls. The Markov chain is used to analyze queuing theory which operates on memory less system, using steady state transition diagram. Also the traffic data are collected from the OMC-counter, which is inbuilt in mobile network.

\subsection{Model Description}

Let us consider a cell system that has some certain volume of offered load in erlang (A) and number of traffic channels. These traffic channels in a cell are partition into two, as follows.

The $\mathrm{V}$ indicates the number of channels use for voice calls within the cell and handover calls. While, g indicates number of channels use for handover calls only. The voice calls and handover calls both use $\mathrm{V}$ and $\mathrm{g}$, having a preemptive priority over data service on these channels. The two types of channels can be access by data service based on availability of these channels. The arrival voice calls and handover voice calls are based on first-come, first served (FCFS) using the TDMA technique.

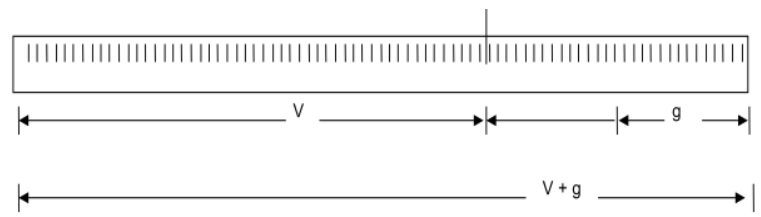

Fig. 3: Channels fragmentation 
The $\mathrm{V}$ indicates the numbers of channels, dedicated for voice calls within the cell and handover calls. While, g represent the number of channels dedicated for handover calls only. The total number of channels in the system is $\mathrm{V}+\mathrm{g}$ for voice calls and handover calls, while these channels have a preemptive priority over the data service based on availability of the channels.

The voice arrival rate $\lambda$ is assumed to follow a Poisson process and the service time (duration of calls) is an exponentially distributed random variable with mean $(1 / \mu)$. Also, we assume that incoming handover calls request, follow a Poisson process, with arrival rate is $\lambda_{h}\left(\lambda_{h}\right.$ is derived by balancing the incoming and outgoing calls handover flows).

The total arrival rate in the cell is $\lambda=\lambda_{\mathrm{V}}+\lambda_{\mathrm{g}}$

$\lambda_{\mathrm{V}}=$ New voice call arrival rate

$\lambda_{\mathrm{g}}=$ Handover call arrival rate

The time spent by a subscriber within a cell (which is normally called dwell time or channel holding time) is assumed to be exponentially distributed with mean $\left(1 / \mu_{\mathrm{h}}\right)$. The call activity time within a network is (call holding time) also a random variable with exponential distribution $\mu_{\mathrm{h}}$.

The total offered traffic in erlang A for voice call is

$$
\begin{aligned}
& A=A_{V}+A_{g} \\
& A_{V}=\text { New voice call offered traffic in erlang } \\
& A_{g}=\text { Handover call offered traffic in erlang } \\
& \therefore A v=\frac{\lambda V}{\mu h} \quad A g=\frac{\lambda g}{\mu g}
\end{aligned}
$$

One dimensional steady state transition diagrams and the application of cut equation are used to express the corresponding state probabilities equation in term of $\mathrm{P}(\mathrm{o})$.

Lets consider, two possible state of $\mathrm{k}$, where $\mathrm{K}$ is a discrete random process representing the number of occupied or busy channels at discrete time. While $\mathrm{P}(\mathrm{K})$ is the probability of observing the system in state $(\mathrm{k})$.

And it as follows:

$P(k)\left\{\begin{array}{rrr}\left(\frac{\lambda}{\mu}\right)^{k} \frac{1}{k !} \cdot P(0) & 0 \leq k \leq V \\ \left(\frac{\lambda}{V \mu}\right)^{k} \frac{1}{(k-1) !} \cdot P(0) & V<k \leq(V+g)\end{array}\right.$

To obtained normalization Equation of the state probabilities for $\mathrm{P}(0)$.

$$
1=\sum_{k=0}^{\infty} P_{K}-
$$

The probability that all the channels are busy may be represented by a fraction of block calls, which is represented by blocking probability $\left(\mathrm{P}_{\mathrm{b}}\right)$.

$$
P_{b}=\frac{\frac{\left(\frac{\lambda}{\mu}\right)^{V}}{V !}}{\sum_{k=0}^{V-1}\left(\frac{\lambda}{\mu}\right)^{k} \frac{1}{k !}+\sum_{k=V}^{V+g}\left(\frac{\lambda}{V \mu}\right)^{k} \frac{1}{(k-1) !}}
$$

The failure of handover call is determine using handover blocking probability $\left(\mathrm{P}_{\mathrm{hf}}\right)$.

$$
\begin{gathered}
P_{n b}= \\
\frac{\left.\frac{(\lambda}{\mu}\right)^{V+g}}{(V+g) !} \\
\sum_{k=0}^{V-1}\left(\frac{\lambda}{\mu}\right)^{k} \frac{1}{k !}+\sum_{k=V}^{V+g}\left(\frac{\lambda}{V \mu}\right)^{k} \frac{1}{(k-1) !}
\end{gathered}
$$

This is experienced when all the $(\mathrm{V}+\mathrm{g})$ channels are busy.

\section{RESULT ANALYSIS AND DISCUSSION OF TRAFFIC MODELS.}

Validation is a quality assurance process of establishing evidence that provides a high degree of assurance that a product, service, or system accomplishes it intended requirements (Wikipedia, 2008). The accuracy of the developed traffic models in Equation 3 and 4 are validated, by using programs written in MATLAB with version 7.6.3 325(2008a). Various scenarios are experimented from low values to higher values of both traffic load in erlang and number of channels $(\mathrm{V})$ to ensure accuracy in determination of the blocking probability traffic model in Equation 3 and handover blocking probability in Equation 4. The total number of channels is divided into two segments $(\mathrm{V}+\mathrm{g})$ traffic channels.

The first scenario, from Equation 3, it's the blocking probability experiences in a certain number of transmission channels $(\mathrm{V})$ only in the mobile network. The $\mathrm{V}$ represents the number of channels used for calls (subscribers) within the cell and handover calls process.

The input parameters such as $\mathrm{V}, \mathrm{g}$ and $\mathrm{A}$ the offered traffic in erlang are substituted into MATLAB program environment. The total number of channels per cell is assumed to range between 1 to 100 and the offered traffic load in erlang (A) values are obtained from the field (Globacom Network) to determine the blocking probability.

Firstly, low number of channels $(\mathrm{V}=7,8,9,10 \& 11)$ are used in MATLAB program environment to ensure accuracy of the simulation result, these values are used to determine the blocking probability against offered traffic load in erlang(A), showed in Fig 4.

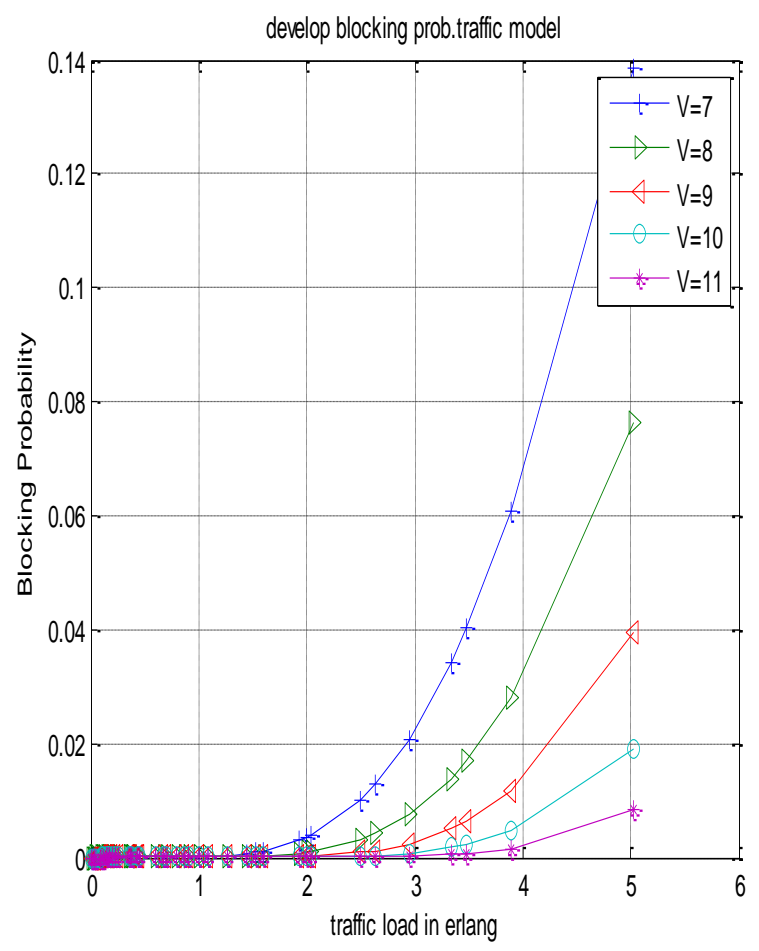

Fig 4 Calls blocking probability against offered traffic load (A) with low number of channels $(V)$. 
In Fig 3 and Fig 4, it is observed that teletraffic system such as the one investigated,exhibit non-linear charecteristics.It is observed that, as the value of offered traffic load in erlang increases the blocking probability valves also increases with a given number of channels $(\mathrm{V})$.At low offered traffic load in erlang,the non-linear charecteristics experienced a linear path, with low blocking probability values. This implies that at low values of offered traffic load in erlang in the network, the resultant blocking probability (block calls) experience in the network is low. Thereafter, the curve line begin to increase in the corresponding blocking probability values and offered traffic load in erlang with a given number of channels (V). The blocking probability is highly influenced by the function of offered traffic load in erlang and the number of channels in the system. For a given number of channels, the system performance increases as the number of traffic load in erlang decreases and (vice- versa).

When a low number of channels $(\mathrm{V})$ are used in the network, it's liable to experience high value of blocking probability with high proportion of offered traffic load in erlang. Fig 4 shows only low number of channels (V) and Fig 5 shows high number of channels (V).

Fig 6 Calls blocking probability with high number of channels (V)

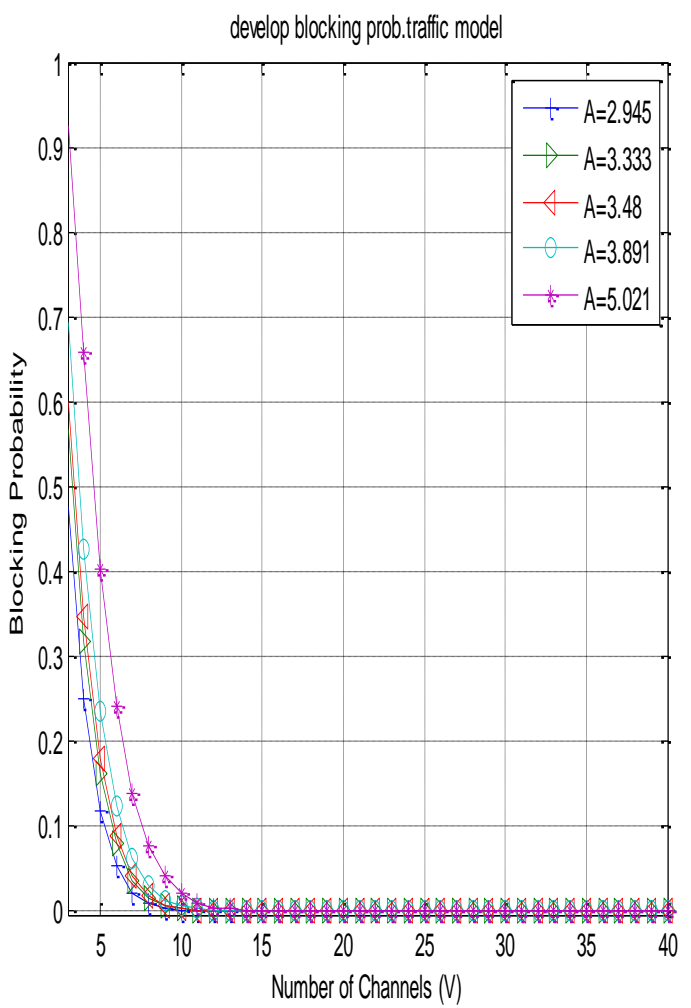

Fig 7 Calls blocking probability against number of channels $(V)$ with simulation of low values of offered traffic load (A).

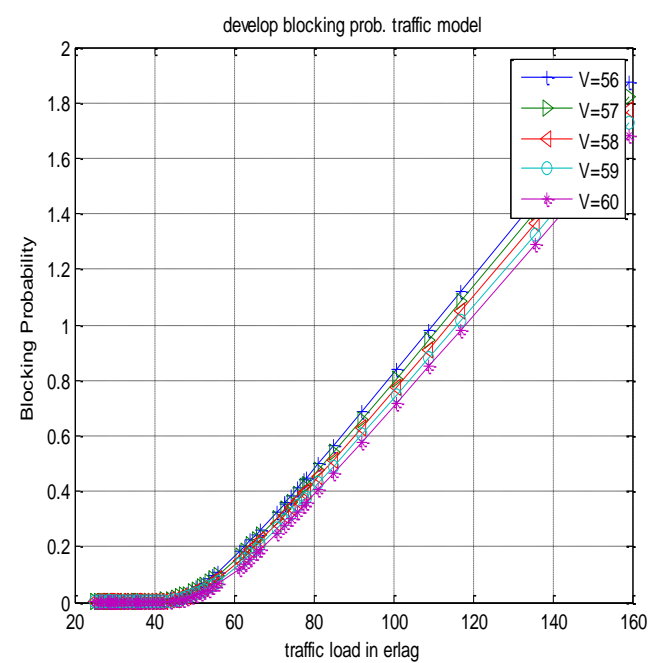

Fig 8 calls blocking probability against offered traffic load in erlang with simulation of high values of number of channels $(\mathbf{V})$.

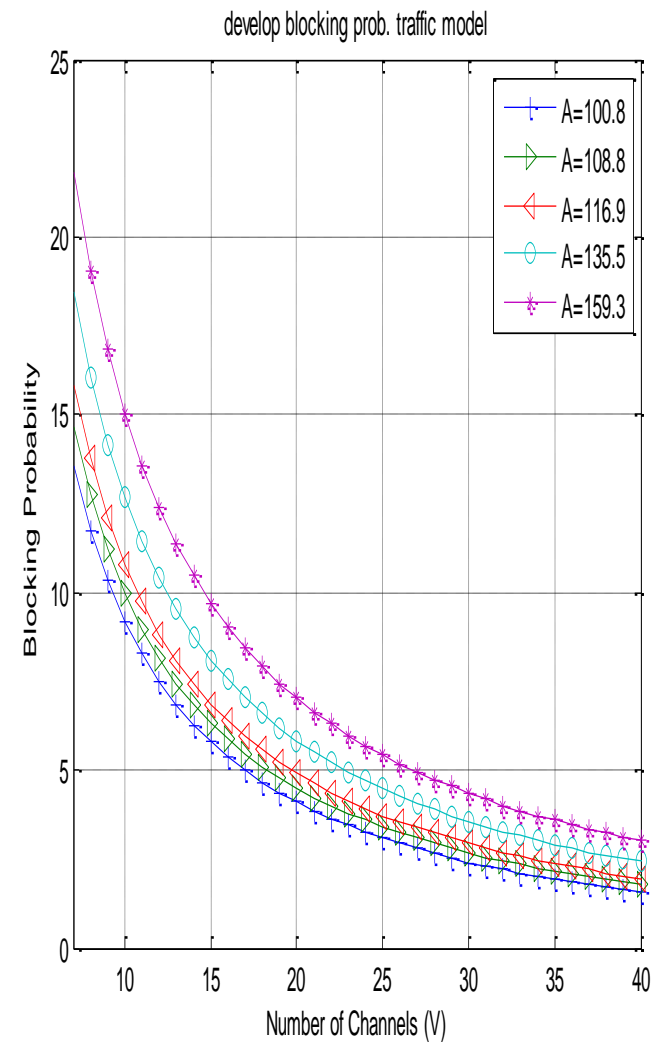

Fig 8 Calls blocking probability against number of channels $(V)$ with simulation of high values of offered traffic load (A). 
Fig 7 and Fig 8, show a MATLAB simulation result obtained with a certain range of number of offered traffic load in erlang. In Fig 7, low number of offered traffic load in erlang, Fig 8 high number of offered traffic load in erlang were used to determine the blocking probability with increasing number of channels (V). It is observed that, there are high value of blocking probability at low number of channels at constant offered traffic load in erlang and also, as the number of channels increases at constant offered traffic load, the blocking probability decreases. Therefore as the number of channels (V) increases, the blocking probability experience in the mobile network decreases. The blocking probability is influenced by the number of channels and offered traffic load in the system. Blocking probability is a major parameter used for evaluating good Quality of Service (QOS).Therefore, for improved QOS, the blocking probability must be minimized to a certain level ( $2 \%$ recommend by NCC) to grantee good QOS in mobile network. A certain value of blocking probability has a corresponding number of channels $(\mathrm{V})$ and offered traffic load in erlang. Therefore to ensure minimized blocking probability value the number of channels and offered traffic in erlang must be adjusted to a suitable desirable level in the network

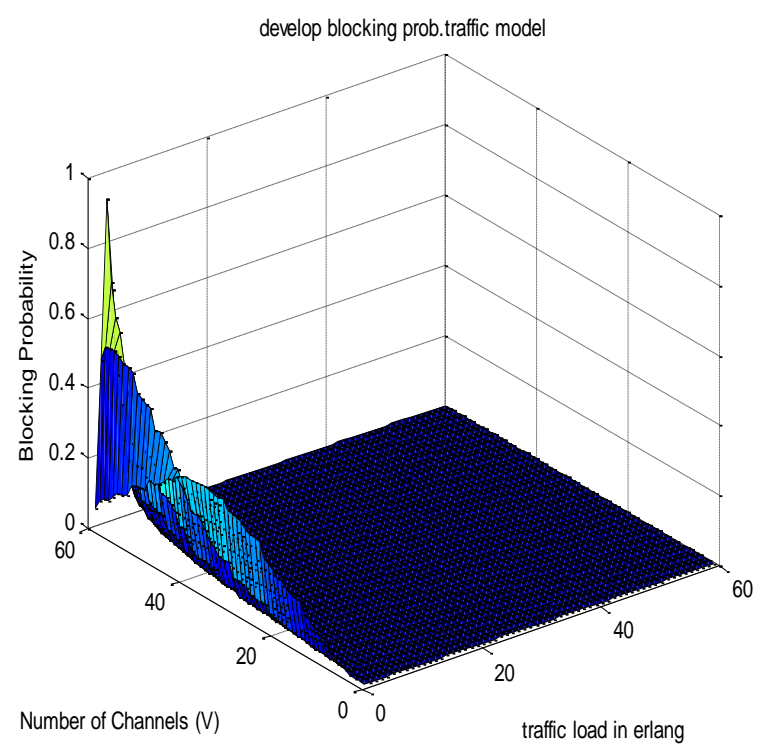

Fig 9 3D calls blocking probability

Fig 9 shows a 3D graph, which indicates that blocking probability $\left(\mathrm{P}_{\mathrm{b}}\right)$ on $\mathrm{Z}$-axis is dependent on number of channels (V) on y-axis and on offered traffic load in Erlang (A) on xaxis. Therefore, blocking probability is a function of number of channels and traffic load in erlang $\left(\mathrm{P}_{\mathrm{b}}=\mathrm{f}(\mathrm{A}, \mathrm{V})\right)$. The traffic model performance of system is based on decrease the blocking probability, as the number of channels (V) increases at a constant traffic load in erlang. In order to obtain satisfactory blocking probability the number of channels must be increased, in a proportion to traffic load in erlang.

\subsection{Handover Blocking Probability Traffic Model}

The second scenario is based on handover traffic model; in Equation 4.This handover propose traffic model is developed based on the unique future (such as mobility of subscribers) associated with mobile communication. The mobile communication subscribers often experience block calls (handover failure calls or handover dropping calls) during the handover process in the network. Therefore, there is need to evaluate the handover block calls experienced by the subscribers during the handover process, using Equation 4 and try to minimized the number of block calls experience during handover process. This handover traffic model Equation is design based on assumptions that this total number of channels $(\mathrm{V}+\mathrm{g})$ are used by handover calls, are exponentially distributed and out going handover calls is equal to in coming handover calls. The number of channels $\mathrm{V}$ are used by calls within the cell and handover calls, while the number of channels $g$ are reserved for handover calls only.

The Equation 4 is validated using the MATLAB program with low input number of channels parameters used in Fig 10 and high input number of channels parameters is used in Fig 11, for the purpose of accuracy.

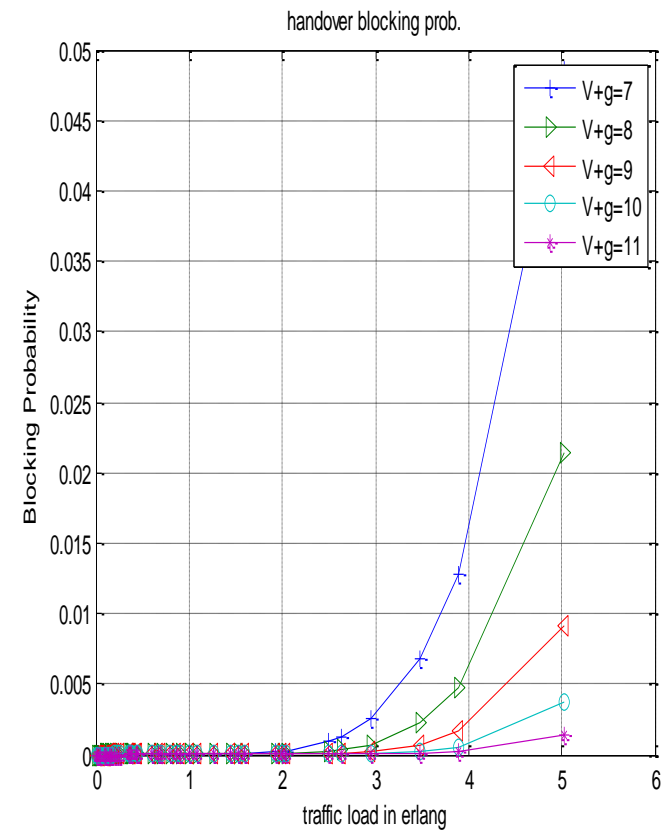

Fig 10 calls handover blocking probability with low number of channels $(\mathrm{V}+\mathrm{g})$.

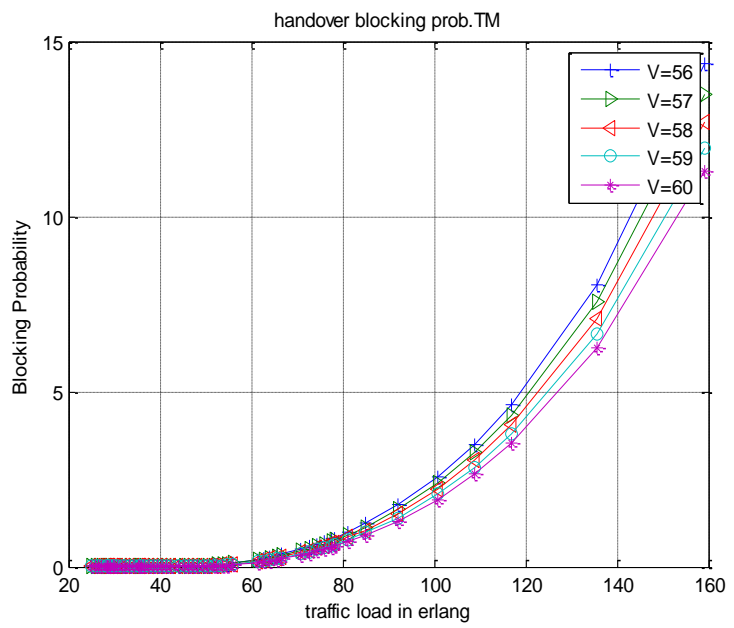

Fig 11 calls handover blocking probability with high number of channels $(\mathrm{V}+\mathrm{g})$. 
It is observed that handover blocking probability is increasing as the handover number of offered traffic load in erlang increases at constant number of channels $(\mathrm{V}+\mathrm{g})$ for Fig 10 and Fig 11.

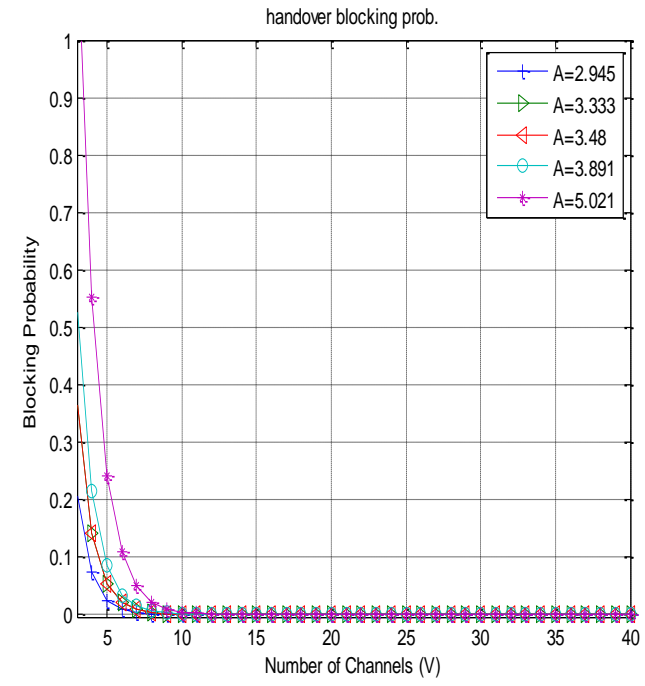

Fig 12 calls handover blocking probability with low offered traffic load in erlang(A).

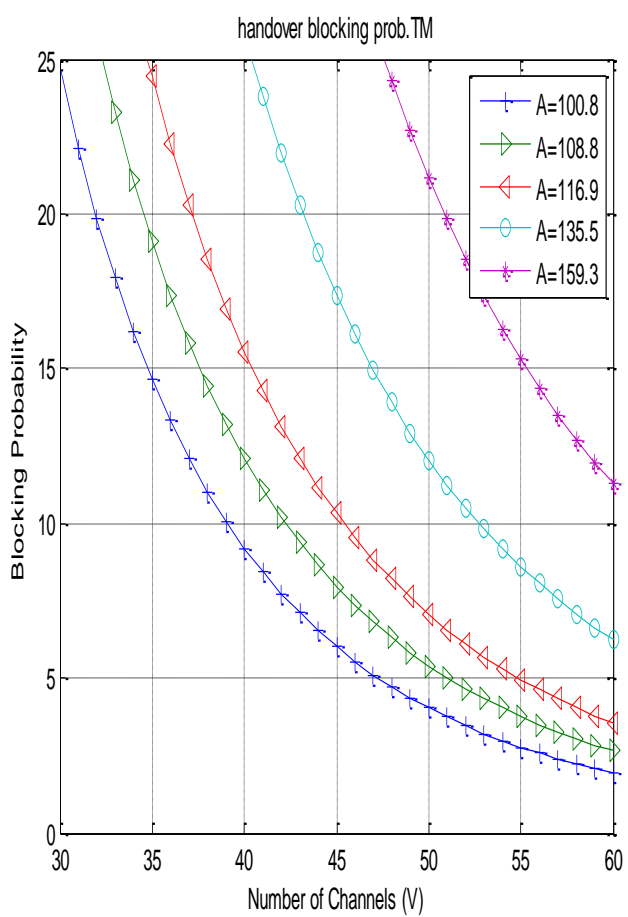

Fig 13 calls handover blocking probability with high number of traffic load in erlang(A).

In Fig 12 and Fig 13, a low traffic load in erlang and a high offered traffic load in erlang are used respectively in determination of the handover blocking probability with an increasing number of channels. It is observed that at constant offered traffic load in erlang (A), the system experienced, high number of handover blocking probability before dropping as the number of channels in the system increases.

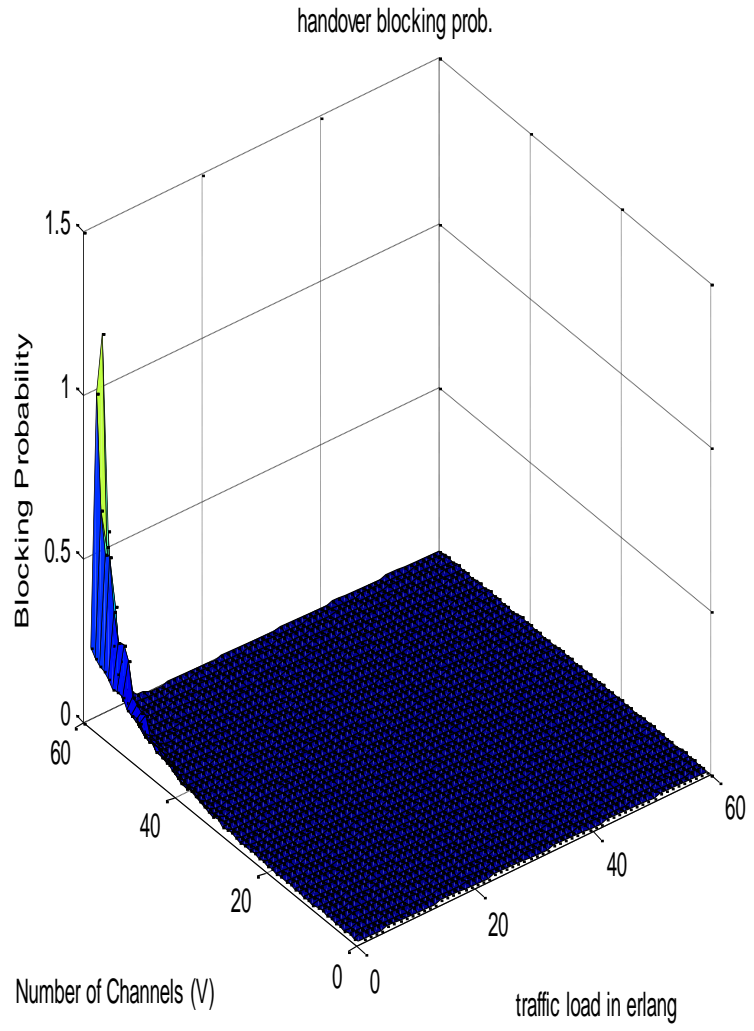

Fig 14 3D calls handover blocking probability.

In Fig 14, the 3D graph shows that handover blocking probability $\left(\mathrm{P}_{\mathrm{b}}\right)$ on $\mathrm{Z}$-axis is dependent on number of channels $(\mathrm{V}+\mathrm{g})$ on $\mathrm{y}$-axis and on offered traffic load in Erlang $(\mathrm{A})$ on $\mathrm{x}$ axis. The handover traffic model performance of the system is based on function of handover blocking probability $\left(\mathrm{P}_{\mathrm{b}}=\mathrm{f}(\mathrm{A},(\mathrm{V}+\mathrm{g}))\right.$. The handover blocking probability decreases as the number of channels $(\mathrm{V}+\mathrm{g})$ and traffic load in erlang increases. In order, to obtain satisfactory handover blocking probability the number of channels must be increased in a proportion to offered traffic load in erlang.

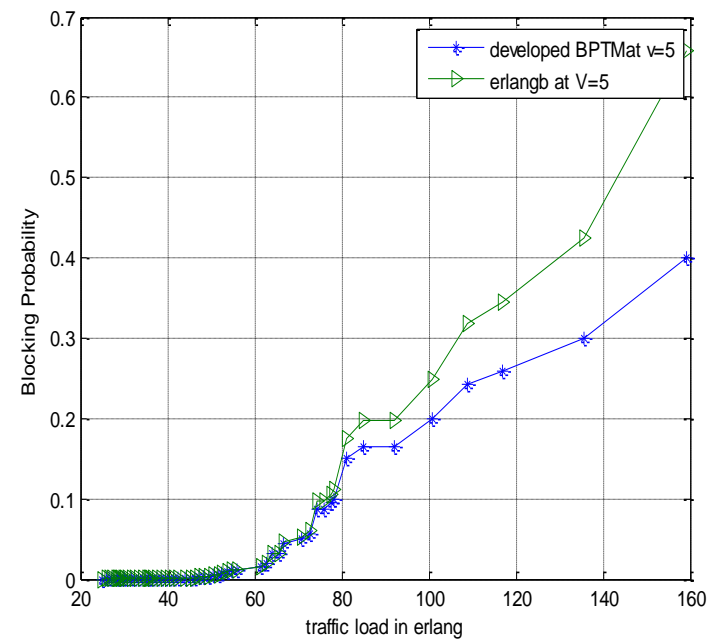

Fig 15a comparison of blocking probability traffic models between Erlang B and developed model (using low number of channels for simulation). 


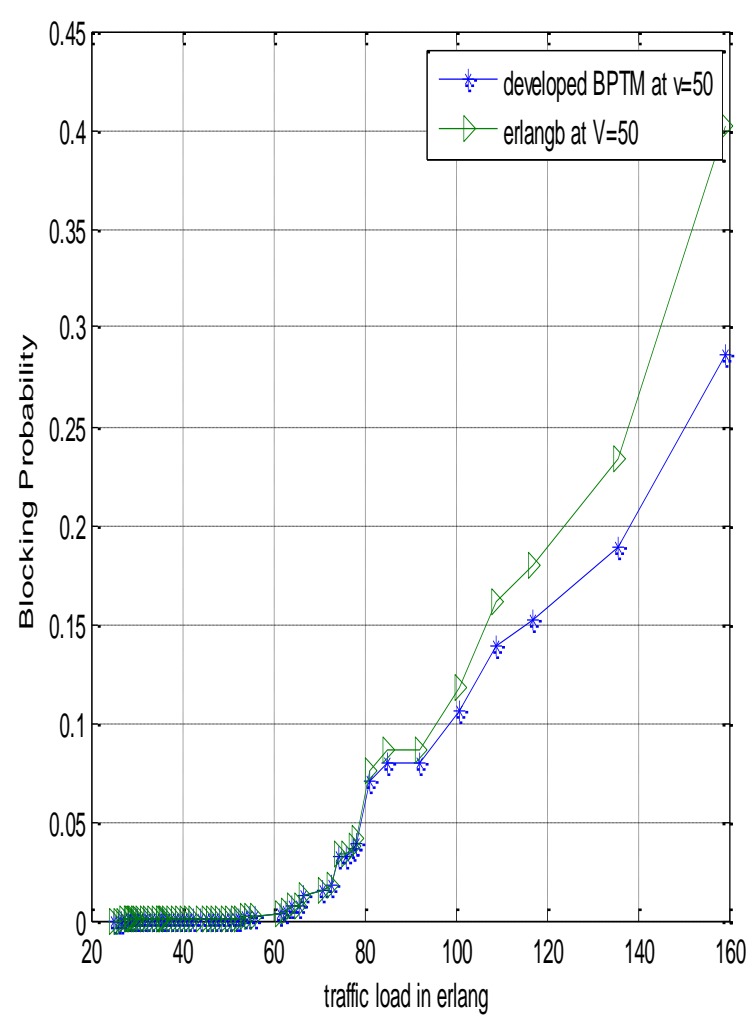

Fig 15b comparison of blocking probability offered traffic models between Erlang B and developed model (using high number of channels for simulation).

The accuracy of the developed traffic model in Equation 3 is compared with the prevailing Erlang B traffic model. The compared traffic models results are shown in Fig 15a and Fig15b using the same input parameters values, both low number of channels and high number of channels to ensure accuracy in the MATLAB program. It is observed in both Figs $15 \mathrm{a}$ and $15 \mathrm{~b}$, that the line graph shows no significant variation due to less offered traffic load, until when the offered traffic load in erlang exceeds some certain range. This variation experienced between Erlang $\mathrm{B}$ and developed (propose) traffic model is due to the number of channels $\mathrm{g}$ reserved for handover calls only.

Also, as the offered traffic load in erlang increases, the blocking probability increases at a constant number of channels V. It is observed, that the developed (propose) traffic model has lower blocking probability compared to Erlang B, this is based on the extra number of traffic channels reserved for handover process.

The significance of these traffic models (the Erlang B, developed traffic model etc) are used to determined the number of channels required to carried or serve a certain traffic load in erlang in a particular geographical location (example are campus, highway, residential area, industrial estate etc) within a recommended (2\%) blocking probability. The effect of high blocking probability, will leads to poor Quality of Service (QOS) resulting in high block calls experience in mobile communication network. Zero blocking probability, will also leads to wasteful of expensive channels (resources) in mobile communication network. Therefore, traffic model is used to manage, a balance relationship between cost incurred by mobile communication operators and service render to the mobile subscribers.

\section{CONCLUSION}

The analytical method is deployed in the developed traffic blocking probability models using queue theory based on Markov transition steady state diagram. These traffic models are designed on these following assumptions, that arrival rate follow a Poisson process ,service times is exponentially distributed, blocked calls are cleared, no calls are blocked when any channels is idle. The $\mathrm{V}$ numbers of channels are served on other of First Come First Serve (FCFS) and the numbers of channels are shared into two segments, $\mathrm{V}$ and $\mathrm{g}$ .Where $\mathrm{V}$ the number of channels used by both calls in the cell and handover calls. While, g number of channels are reserved for handover calls only. The data service traffic model is determined, from $\mathrm{V}+\mathrm{g}$ number of channels used for both new voice calls and handover calls having preemptive priority over data service.These developed traffic models are validated using MATLAB program with different range of value, for both number of channels and the traffic load in erlang are used to ensure accuracy .It was observed that the blocking probability function depended on the number of channels and the traffic load in erlang.Therefore, the blocking probability decreases as the number of channels increases at a constant traffic load in erlang. The blocking probability decreases as the traffic load in erlang reduces at constant number of channels. The developed blocking probability was compared with existing Erlang B traffic model; it was observed that the line graph shows no significant variation within a less traffic load, until when the traffic load in erlang exceeds some certain range. This variation experienced between Erlang B and developed traffic model is due to the number of channels $\mathrm{g}$ reserved for handover calls only. The significant of these traffic models (the Erlang B, developed traffic model etc) are used to determined the number of channels required to carried or serve a certain traffic load in erlang in a particular geographical location (example are campus, highway, residential area, industrial estate etc) within a recommended (2\%) blocking probability. Therefore, traffic model is used to manage, a balance relationship between cost incurred in mobile communication by operators and service render to the mobile subscribers.

\section{REFERENCES}

[1] Alexei B., Placido R.P, Alvaro S.N, Ruddy P.P., Rebecca F.P. (2009)“A Novel model for optimized GSM Network Design”, int. journal of computer sci., Vol. 4 NO.1\&2 ,PP 44-48

[2] Cornel B.,Georgeta B., Adrian B,(2010) "Mixed traffic models for Dimensioning Radio Resources in GSM/GPRS Networks" Wseas Transaction on communications,ISSN:1109-2777,Issue 3,Volume 9,pp 233-242

[3] Georgeta, B., Cornel, B., Adrian, B. and Eugen, M. (2009). "Traffic models and Associated parameters in GSM/(E)GPRS Networks" Wseas Transaction on Communications,ISSN:1109-2742,Issue 8,Volume 8,pp 833-841

[4] Gunner, H. (1998). 'GSM Network' $1^{\text {st }}$ Edition Artech House Boston page 217-225, 361-372. 
[5] Houda, K., Fabrice, V. and Sami, T. (2002). Traffic characterization for mobile network. http://www.supcom.mincom.tn/mediation/ articles/62302pdf IEEE page 1-3.

[6] ITU-D (2006). "Teletraffic Engineering" International Telecommunication union http://www.itu.int page 68$69,119-186$.

[7] Jahangir H. S.,Seppo J.H and Mika R(2000) "performance Analysis of GSM traffic channel capacity with(out)high speed circuit switched data" ,IEEE Semiannual Vehicular Technology Conference pp16031609.

[8] Madhusmita P. and Saraju P.P (2011) "Traffic Analysis and optimization of GSM Network "IJCSI Int.journal of computer sci. Issues, Special Issue,ICVCI-2010 ,ISSN:1694-0814

[9] Marco A.M, Paola L.and Michela M.(2003) "Packet Delay Analysis in GPRS Systems" IEEE Journal pp 1-6

[10] Neidhardt A.,Jerkins J. and Krishnan K.R (2008) "Analytical model for evaluating blocking in GSM network with directed retry" http://www.argreanhouse.com/paper/j/j/itcss-wireless pdf.

[11] Rappaport S.T. (2003) wireless communication principles and practice, $2^{\text {nd }}$ Edition,prentice Hall of india private limited, new Delhi,page 35-62.
[12] Singh L.K and Srivastava R. (2007) "Memory estimation of internet server using queuing theory:comparative study between $\mathrm{M} / \mathrm{G} / 1, \mathrm{G} / \mathrm{M} / 1 \& \mathrm{G} / \mathrm{G} / 1$ queuing model" world academy of science, engineering and Technology 33 page 153-157.

[13] Sharma (2010) "Operation Research Theory and application" $4^{\text {th }}$ Edition, Macmillan publishers India Ltd, pp 45-82

[14] Stefano B.,Roberto C.,and Maurizio D. (2008)“An Empirical study on time -correlation of GSM telephone traffic"IEEE Transactions on wireless communications,Vol. 7,No.9,pp3428-3435.

[15] Wikipedia ,(2008) "Verification and validation" the free encyclopedia http:en.wikipedia.org/wiki/IG

[16] Yuguang Fang (2005)"Modeling and Performance Analysis for wireless mobile networks:A New Analytical Approach" IEEE/ACM Transactions on Networking,Vol. 13,NO. 5,PP 989-1002.

[17] Zukerman Moshe (2010) "Introduction to queue theory and stochastic teletraffic model"pp 45-67,87,104-127. http://www.ee city u.edu.hk/Zukerman/classnote pdf. 